\title{
La trayectoria intelectual de Manuel Zapata Olivella en los procesos organizativos afrocolombianos $^{1}$
}

\author{
The intellectual trajectory of Manuel Zapata Olivella in \\ Afro-Colombian organizational Processes
}

\author{
Carlos Alberto Valderrama Rentería \\ Universidad Icesi, Cali, Colombia \\ Universidad del Valle, Cali, Colombia \\ pibeson@gmail.com
}

REsumEn: En el presente artículo examino la trayectoria intelectual de Manuel Zapata Olivella en el proceso organizativo afrocolombiano. Sugiero que fue un intelectual comprometido. Me centraré en dos aspectos de su trayectoria. Primero, analizo cómo Manuel Zapata Olivella se relacionó con organizaciones, agrupaciones e intelectuales afrocolombianos para discutir asuntos relacionados con la identidad racial y el racismo en Colombia. Considero que en estas discusiones Manuel Zapata Olivella fue un actor clave que dinamizó la conformación de esferas públicas afrocolombianas. Segundo, estudio los conflictos y contradicciones que surgieron de estas discusiones. Propongo que estas esferas públicas afrocolombianas fueron un terreno ideológico disputado y contestado.

1 Una versión preliminar de este documento fue presentada en el Simposio Internacional Jorge Isaacs dedicado a Manuel Zapata Olivella, "Tras las Huellas de Manuel Zapata Olivella”, organizado por el Centro Virtual Isaacs de la Universidad del Valle, en Cali, Colombia, 2018. 
Palabras Claves: intelectual comprometido, esferas públicas afrocolombianas, identidad negra, racismo.

Авstract: In this article, I examine the intellectual trajectory of Manuel Zapata Olivella in the Afro-Colombian organizational process. I suggest that he was a committed intellectual. I will focus on two aspects of his trajectory. First, I study the way in which Manuel Zapata Olivella connected to Afro-Colombian organizations, groups, and intellectuals to discuss issues related to black identity and racism in Colombia. I believe that Manuel Zapata Olivella was a key factor in during the emergence of the Afro-Colombian public spheres. Second, I study the conflicts and contradictions that emerged from these discussions. I suggest that these Afro-Colombian public spheres were a terrain ideologically disputed and contested.

KEYWORDs: committed intellectual, black public spheres, black identity, racism.

\section{INTRODUCCIÓN}

En el presente artículo examino la trayectoria intelectual de Manuel Zapata Olivella en el proceso organizativo afrocolombiano. No me centraré en su trayectoria literaria. Hay suficiente material bibliográfico especializado que desarrolla con profundidad sus aportes literarios, ensayísticos e investigativos al campo de las humanidades y de las ciencias sociales (Arboleda, Caicedo, Lewis, Prescott, Tillis, Viveros). No obstante, hay muy poca o nula discusión acerca de sus aportes a los procesos organizativos. De hecho, se ha negado alguna incidencia en el plano de lucha social y política (Wagbou et al. 96). El objetivo del presente artículo es analizar la trayectoria intelectual de Manuel Zapata Olivella en los procesos de movilización social y política afrocolombianos. Mi premisa es que Manuel Zapata Olivella fue un intelectual comprometido que no se quedó simplemente enfocado en la producción de literatura revolucionaria o de combate, sino que también avanzó en acciones concretas que favorecieron la organización social y política contra la opresión en Colombia.

Para lograr demostrar lo anterior, parto del concepto de "contrapúblicos subalternos", entendido como "arenas discursivas paralelas donde los miembros de los grupos subordinados inventan y circularon 
contradiscursos, que a su vez les permite formular interpretaciones de oposición sobre sus identidades, intereses y necesidades" (Fraser 67). A diferencia del concepto de "movimientos sociales", usado con frecuencia para estudiar las movilizaciones sociales afrocolombianas, la noción de "contrapúblicos" me permite identificar aquellos espacios, lugares y formas de la política negra que tuvieron un carácter flexible, que estuvieron estructurados sobre la base de relaciones e interacciones sociales, que presentaron una estructura amorfa dada su flexibilidad para articular diferentes esferas públicas culturales, sociales, y políticas, y que son un campo de disputas discursivas debido a la presencia de diferentes agendas políticas y sujetos y sujetas afrocolombianas con diferentes subjetividades políticas.

Utilizo este concepto para identificar aquellas esferas públicas afrocolombianas con las que Manuel Zapata Olivella se relacionó, se movilizó y/o discutió asuntos relacionados con la identidad negra y el racismo en Colombia. Estas interacciones muestran la manera en que Manuel Zapata Olivella participó en la emergencia, desarrollo y consolidación de una forma organizativa que, a diferencia de otras esferas públicas en las que él participó -como grupos de izquierda, liberales o sociedades de café-, contribuyeron en la constitución de un contrapúblico afrocolombiano autónomo e independiente del mercado, el Estado u otras organizaciones. Estas esferas públicas afrocolombianas permitieron, entonces, que miembros racialmente subordinados discutieran cuestiones de raza, racismo e identidad negra/ afro para definir sus intereses, necesidades y agendas, como explica Michael Dawson en su obra Black Visions: The Roots of Contemporary African-American Political Ideologies. En este orden de ideas, ¿cómo la trayectoria intelectual de Manuel Zapata Olivella nos revela su participación en la conformación de este contrapúblico afrocolombiano? Para responder a esta interrogante, analicé sus modalidades de participación y activismo desde el periodo de 1943 -cuando inició su participación social en procesos organizativos- hasta finales de los setenta, cuando impulsó la consolidación del movimiento de la negritud. Es un periodo en el que Manuel Zapata Olivella va de lo local a lo global en su construcción, no solo de una conciencia racial afrocolombiana, sino también de redes transnacionales afrodiaspóricas. 
La estrategia metodológica fue la siguiente. Primero, revisé la literatura disponible sobre la vida, obra y trayectoria de Manuel Zapata Olivella. Segundo, realicé conversaciones con activistas, bailarines, músicos, intelectuales y académicos que se relacionaron con Manuel Zapata Olivella y participaron de actividades, conferencias y tertulias en el periodo mencionado. Y, tercero, partiendo de las conversaciones realizadas pude identificar fechas y eventos que consideré claves para mi ejercicio de revisión documental de periódicos de tiraje nacional -como El Espectador y El Tiempo-y local -como El País de Cali-. La perspectiva del escrito es histórica. Presento un resumen de las relaciones que Manuel Zapata Olivella tuvo con organizaciones, agrupaciones e intelectuales durante la emergencia, desarrollo y consolidación de esferas públicas afrocolombianas. En este sentido, me centré en dos aspectos de su trayectoria. Primero, analizo cómo Manuel Zapata Olivella se relacionó con estas esferas públicas afrocolombianas para discutir sobre asuntos relacionados con la identidad negra y el racismo en Colombia. Lo anterior me permitió sostener que Manuel Zapata Olivella fue un actor clave que ayudó a dinamizar en la consolidación del contrapúblico afrocolombiano. Segundo, los conflictos y contradicciones que surgieron de estas discusiones convirtieron el contrapúblico en un terreno ideológico disputado y contestado.

\section{REDES QUE DESATAN EL NUdO DE LOS COMPLEJOS RACIALES}

Una manera de observar cómo Manuel Zapata Olivella se relacionó con organizaciones, agrupaciones e intelectuales afrocolombianos es analizar las redes de relaciones y colaboraciones que le ayudaron a "desatar el nudo de los complejos raciales inconscientes" (Zapata, Levántate Mulato 184). En la Bogotá de los años cuarenta, jóvenes negros del Pacífico y del Caribe colombiano usaron las pensiones, las aulas de clases y las calles para discutir qué significaba ser negro en Colombia. Así lo cuenta el propio Manuel Zapata Olivella:

[L]os estudiantes costeños del Caribe nos vimos abocados a violentas confrontaciones, evidentes entre nuestra condición de mulatos o zambos y la etnia puramente negra de los condiscípulos caucanos. En esos encuentros capitalinos en pensiones, aulas y 
calles discutíamos, sin saberlo, lo más importante de la formación humanística: nuestra propia identidad. "Tú eres negro", "Yo soy mulato". "Todos somos discriminados" (...) Paulatinamente, dolorosamente, al lado de mi hermana (Delia Zapata) principiamos a desatar el nudo de los complejos raciales inconsciente[s] (ibid.).

Estas, en mi opinión, son las primeras esferas públicas de carácter nacional de las que Manuel Zapata Olivella hizo parte. Como su testimonio lo indica, eran estudiantes afrocolombianos provenientes de varios lugares de Colombia. Entre estos se encontraron el líder político chocoano Diego Luis Córdoba, los líderes políticos norte caucanos Natanael Díaz, Marino Viveros, Adolfo Mina Balanta y Arquímedes Viveros, el poeta y novelista guapireño Helcías Martán Góngora, la folclorista y artista Delia Zapata Olivella, y el médico y ensayista Manuel Zapata Olivella, entre otros. Como se observa en su testimonio, las pensiones, las aulas y las calles fueron los espacios donde se discutió bajo la consigna "Tú eres negro, Yo soy mulato. Todos somos discriminados", al modo de una esfera pública que surgió de manera espontánea, independiente y autónoma de otras esferas ya institucionalizadas para la época. Me refiero con esto último a los partidos políticos, las organizaciones sindicales, sociedades de café, etcétera, que predominaban en la Bogotá de los años cuarenta y en las que muchos de estos afrocolombianos también participaron.

De las pensiones, las aulas y la calle, se pasó a la protesta. Manuel Zapata Olivella salió a participar de las protestas realizadas durante la celebración del Día del Negro. Esta fue una manifestación contra el racismo, realizada por un grupo de jóvenes afrocolombianos el 20 de junio de 1943 en Bogotá. Según lo expresa el mismo Manuel Zapata Olivella, la marcha buscó hacer pública la solidaridad racial de los afrocolombianos con los trabajadores negros linchados en la ciudad de Chicago, Estados Unidos (Zapata, Levántate Mulato 187). En la marcha participaron al menos doce estudiantes negros universitarios, entre los cuales se encontraban aquellos que hicieron parte de las conversaciones en las pensiones, aulas y calles. Las actividades desarrolladas durante la marcha incluyeron:

La presencia de tres a cuatro negros gritando vivas a su raza dentro de las aulas [que] dejaba perplejos a los alumnos. Hasta ese momento, y seguramente después, los mestizos, mulatos, y zambos, y aun los propios negros o indios, jamás se habían cuestionado su identidad étnica. Nos miraban sorprendidos, 
reivindicadores de una causa inexistente. La mayoría se mostraron molestos porque se les evidenciaría los nexos de sangre que poseían con la raza discriminada. (Zapata, Levántate Mulato 188).

Los manifestantes del Día del Negro se dirigieron a la biblioteca de la Universidad Nacional de Colombia, Bogotá, y solicitaron que se reprodujera música de los artistas afroamericanos Marian Anderson y Paul Robeson. Así, exigieron "que en toda la mañana se tocara música de nuestros hermanos de raza. Finalmente se inició el concierto como lo pedíamos y todos en silencio escuchamos durante tres horas los himnos religiosos del pueblo negro norteamericano" (Zapata, Levántate Mulato 188). Por otro lado, caminando por la calle séptima de Bogotá y empuñando sus manos en el aire, los protestantes arengaban, “ ¡Vivan los negros!”, “ ¡Abajo la discriminación racial!", "Protestamos por los linchamientos de nuestros hermanos de raza en los Estados Unidos!” y “¡Viva el África del año dos mil!” (189). Posteriormente, los manifestantes se detuvieron en cafés donde intelectuales blancos y mestizos de la capital solían discutir asuntos nacionales. "Natanael Díaz, fogoso orador, se subía a una mesa y arengaba. El coro de sus compañeros aplaudíamos y cantábamos: ¡Ay, mamá Inés! ¡Ay, mamá Inés! ¡Todos los Negros Tomamos Café!” (189). En el café, los manifestantes recitaron poemas de Candelario Obeso y Jorge Artel, leyeron partes de la novela Sangre Negra -en inglés, Native Son- escrita por Richard Wright, guardaron un minuto de silencio por la muerte de George Washington Carver y tocaron música cumbia y rumba (Pisano 66-67).

La marcha terminó en la noche, cuando el grupo de estudiantes se dirigió a la estatua de Simón Bolívar en Bogotá. Después de expresar algunos agradecimientos, Adolfo Mina Balanta, del norte del Cauca, recriminó a la estatua del libertador por no cumplir su promesa de liberar a los esclavizados después de la independencia. El acto hizo que las autoridades actuaran contra los manifestantes por lo que la recriminación terminó, para Manuel y Delia Zapata Olivella, Natanael Díaz, Adolfo Mina Balanta y Marino Viveros, en una estación de la policía, mientras que otros lograron escapar (Zapata, Levántate Mulato 190; Pisano 67).

De la protesta pasaron a la organización colectiva. Como consecuencia de la celebración del Día del Negro, los manifestantes decidieron crear el Club Negro de Colombia (Pisano 66). Sus miembros fueron los políticos liberales y afrocolombianos del norte del Cauca como Marino A. Viveros, presidente, Natanael Díaz, relacionista público, y Víctor M. Viveros, teso- 
rero; el poeta guapireño y mulato Helcías Martán Góngora, vicepresidente, y Manuel Zapata Olivella, del Caribe colombiano, secretario general. Sus objetivos fueron construir una biblioteca afrocolombiana, edificar un hogar para migrantes afrocolombianos en Bogotá, realizar conferencias sobre las condiciones de la gente negra en la diáspora africana y crear un barrio afrocolombiano similar al barrio afroamericano Harlem en Nueva York, Estados Unidos (Pisano 68). Por otro lado, el Centro de Estudios Afrocolombianos fue creado por Manuel Zapata Olivella en 1947, en Bogotá. Fue una iniciativa cultural y académica en la que participaron intelectuales, políticos, poetas y folcloristas provenientes de la región del Pacífico y del Caribe colombiano ${ }^{2}$. En adelante, podemos observar un papel protagónico del autor en la conformación de la red de relaciones y colaboraciones de la que, en mi opinión, él fue un nodo central. Su creación ocurrió después de una gira hecha por Manuel Zapata Olivella en el sur, centro y norte de América. Este fue el viaje en el que Manuel Zapata Olivella conoció la literatura antillana, afroamericana y africana a través de la influencia del afroamericano Langston Hughes (Prescott, "Brother to Brother" 91). Fue el poeta afrocolombiano Jorge Artel quien le enseñó a Manuel Zapata Olivella la existencia de este intelectual afroamericano (ibid.). En 1947, bajo el nombre de Manuel Karabali, Manuel Zapata Olivella reportó en la revista Cromos que:

$[\mathrm{H}]$ oy en día, con una visión más clara de la realidad del negro en la América, estimulados por los estudios que se adelantan en otros países del continente y convencidos de que la tarea de empezar los estudios sobre la raza era impostergable, los mismos precursores del movimiento han fundado el Centro de Estudios Afro-Colombianos, bajo la protección técnica del Instituto de Etnología, dirigido por el profesor Luis Duque Gómez (Karabalí 9)³.

2 Entre sus miembros estuvieron los afrocolombianos César Alonso, Marino Viveros, Carlos Calderón Mosquera, Delia Zapata Olivella, Baldomero Sanín Cano, Alberto Miramón, Natanael Díaz, Diego Luis Córdoba, Dulcey Vergara, y Guillermo Naneti; y los intelectuales mestizos y blancos, Gregorio Hernández de Alba y Duque Gómez (Karabalí).

3 El Club Negro de Colombia y el Centro de Estudios afrocolombianos tuvieron una corta vida (Pisano). No hay razones ni causas que nos permitan entender por qué fueron organizaciones efímeras. Tampoco hemos podido registrar si hubo otras esferas públicas negras/afrocolombianas que sucedieron a las anteriores. Lo cierto es que con el asesinato del líder Jorge Eliecer Gaitán en 1948, la seguridad física de Manuel Zapata Olivella se vio amenazada. Así lo recuerda en su libro 
Además de la creación del Centro de Estudios Afrocolombianos, Manuel Zapata Olivella mantuvo una estrecha relación con intelectuales negros y afrocolombianos, a quienes, en ocasiones, facilitó recursos para que publicaran sus trabajos literarios. Por ejemplo, en 1948, Arnoldo Palacios conoció a Manuel Zapata Olivella en Bogotá. En ese momento, Manuel Zapata Olivella se encontraba con Gabriel García Márquez y Enrique Buenaventura (Collazos 16). En los acontecimientos del 9 de abril, Arnoldo Palacios perdió el manuscrito de su famosa obra Las estrellas son negras. Según Prescott, fue Manuel Zapata Olivella quien animó a Arnoldo Palacios a reescribir la novela (Without Hatreds or Fears 62) y luego intercedió para que Clemente Airó la publicara (Collazos 17). Años más tarde, en 1953, Manuel Zapata Olivella, como jefe de la Alianza de Escritores Colombianos, apoyó a Rogelio Velásquez con la publicación de su novela Las memorias del odio (Valderrama, The negritude movements 129).

Es muy importante reconocer, en este sentido, cómo se fue conformando el contrapúblico afrocolombiano entre las décadas del cuarenta y el cincuenta. En el panorama que hemos reseñado hasta aquí, podemos observar cómo las esferas públicas de discusión fueron el resultado de interacciones espontáneas entre estudiantes afrocolombianos en Bogotá, provenientes de diferentes regiones. Observamos, también, la producción de literatura con contenidos que reflejan la realidad y experiencia de ser negro en Colombia (Caicedo 17) y, por último, podemos observar el uso que estos intelectuales hicieron de los estudios y las agrupaciones folclóricas para visibilizar lo que la nación, el mestizaje y el indigenismo institucional pretendieron ocultar: la existencia de una cultura negra en Colombia. También hay que reconocer que gran parte de las esferas que se consolidaron en la literatura y en los estudios folclóricos fueron influenciadas por corrientes de pensamiento como el negrismo cubano y los estudios americanistas (Valderrama, The negritude movements 132).

Levántate Mulato!: "Egresado de la Universidad, perseguido por mis ideas y militancia en pro de la revolución social, mi vida en la capital de la República ya se contaba entre las sepulturas del cementerio. Algunos primos residentes en la frontera con Venezuela me brindaron refugio en la provincia del Valle de Upar. Elegí los límites de la patria porque me permitía rechazar la idea del auto-exilio y seguir asumiendo un puesto de lucha junto ami pueblo" (288). 
En este sentido, el folclore fue una de las dimensiones del trabajo intelectual que Manuel Zapata Olivella ayudó a desarrollar entre 1960 y 1970. Según he podido demostrar en mis estudios, la red de colaboración implicó apoyos para el estudio, recopilación y montaje de prácticas tradicionales afrocolombianas en danzas coreográficas (The negritude movements 189-200). Manuel Zapata Olivella entendió que la sabiduría y conocimiento de los mayores, habitantes de zona rural y campesinos, fueron fundamentales en la reconstrucción histórica no solo de la danza, los instrumentos musicales y el vestuario, sino también de las particularidades de cada región de Colombia. Para acceder a estos conocimientos y sabiduría de los mayores de cada región, Manuel Zapata Olivella se valió de la red de relaciones y colaboraciones que desarrolló con Teófilo Potes y Mercedes Montańo, en Buenaventura; y Teresa Martínez de Varela, Antero Agualimpia, Rogelio Velásquez y Madolia de Diego, en Chocó. Este último participó, además, en las agrupaciones folclóricas de Delia Zapata Olivella. Teresa Martínez fue muy importante en el reclutamiento de bailarines. En el norte del Cauca, tuvo contacto con Leonor y Raúl Gonzales Mina y Alonso Sandoval, de los Macheteros del Cauca. En el Caribe colombiano, se acercó a Aquiles Escalante, Batata I, Los gaiteros de San Jacinto, Rafael Escalona, Jorge Artel, Orlando Fals Borda, etcétera (Valderrama, The negritude movements 189).

Creo que estas relaciones son ejemplo de la manera en que Manuel Zapata Olivella fue consolidando su red de apoyo, en la que se movilizaron e intercambiaron ideas, proyectos, agendas e intereses. No podemos olvidar que algunos de quienes conformaron sus primeros grupos folclóricos fueron campesinos, labriegos, pescadores o personas que, para ese entonces, no tenían el reconocimiento que hoy tienen en el campo folclórico y cultural. Algunos de ellos fueron Lorenzo Miranda, Batata y los Gaiteros de San Jacinto, en el Atlántico; y Toto la Mompoxina, Leonor Gonzales Mina, Madolia de Diego y Esteban Cabezas, en la región Pacífica. Delia y Manuel no solo viajaron por toda Colombia mostrando la música, danzas, vestuarios e instrumentos musicales de estas regiones, sino que también lo hicieron en Europa y el sur de América. Comparto con Viveros que es necesario reconocer los aportes que hicieron los hermanos Zapata Olivella por la difusión de la tradición musical y dancística de los sectores populares y afrocolombianas, no solo desconocida sino también desvalorada como música vulgar o "música de negros" (Viveros 460). Un aspecto en general poco destacado es que las 
danzas coreográficas fueron en sí mismas el medio utilizado para dar a conocer las formas de vida locales de estos grupos étnicos y raciales. Para Delia y Manuel Zapata Olivella, las danzas y producciones coreográficas eran "estampas culturales", ya que ellas teatralizaron la vida cotidiana de campesinos afrocolombianos e indígenas. Así, proyectaron aspectos de la vida local, tales como la música e instrumentos musicales, la tradición oral (cuentos, leyendas, mitos, décimas, cantos, etcétera), la religiosidad (adoraciones, alabaos, arrullos, chigualos, etcétera), los bailes (de laboreo, profanas, de coqueteo, etcétera) y una estética cultural en el vestuario y peinados. Estudiaron y montaron danzas coreográficas populares no solo por estética o esnobismo. Observaron una agenda folclórica que interpeló a la nación mestiza que negaba la existencia de lo negro en el país.

A partir de todo lo anterior, podemos señalar que Manuel Zapata Olivella fue uno de los pioneros en los estudios y divulgación de la cultura y la historia nacional colombiana, que incluyó un énfasis en los aportes culturales de la gente negra a ella, en un momento histórico en que la mayoría de los investigadores colombianos en el campo de la historia y la antropología los invisibilizaron (De Friedemann 509) y se negaron a realizar investigaciones sobre las culturas e historias afrocolombianas y su relación con África, la trata esclavista, la explotación y la discriminación racial. El activismo cultural de Manuel Zapata Olivella y su hermana les permitió ampliar esta red de relaciones y colaboraciones con la creación de la Fundación Colombiana de Investigaciones Folclóricas, la revista Letras Nacionales, la creación y emisión de programas folclóricos en la radiodifusora nacional de Colombia, la creación del Teatro Anónimo Identificador-similar al Teatro del oprimido-, con el que recorrió varias plazas y parques de Colombia presentando obras como Rambao; y la reapertura del Centro de Estudios Afrocolombianos (Valderrama, The negritude movements 167-220). 


\section{De TERTUliAs AL PRIMER CONGRESO DE CULTURA NEGRA}

El número de estudiantes afrodescendientes en las principales ciudades colombianas se incrementó sustantivamente y con ellos las tertulias y/o encuentros fueron tomando fuerza en centros urbanos como Bogotá, Cali, Popayán, Pereira, Barranquilla y Medellín. Según pude constatar en conversaciones con activistas, líderes y lideresas del movimiento social afrocolombiano, las tertulias y/o encuentros entre estudiantes de diferentes lugares de Colombia se iniciaron por diversas razones. En muchos casos, se trató de un espacio de encuentro y de esparcimiento, mientras que en otros el detonante fue el racismo vivido por afrocolombianos en las ciudades. Así describe Juan de Dios Mosquera, líder del movimiento Cimarrón en Colombia, la situación:

Eran $[\ldots]$ estudiantes porque eran los que comenzaban a pensar y sufrían el racismo en el interior del país. No surge en Buenaventura, Tumaco, Quibdó, no surge ni en Cartagena sino acá donde el otro me hace sentir el otro. Mira la historia de cómo comienza Soweto en Pereira donde te hacen sentir el otro cuando te dicen negro y te dicen cuscú y te dicen esto. "Negro cuscú", un concepto de la costa atlántica que utilizaban los paisas para decirnos negros (Conversación. Juan de Dios Mosquera. Bogotá, 2015).

Lo anterior es parte de la historia que llevó al surgimiento del colectivo Soweto en Pereira a inicios de la década del setenta. Pero similar dinámica se desarrolló con el Frente Amplio por la Liberación del Negro Colombiano, en la Universidad del Valle (Angulo 15-40) el colectivo de estudiantes en la universidad del Cauca, liderado por Eliecer Hurtado, Aura Rosmira, Stella Vida y el hijo de Juan Zapata, Alexis Zapata Olivella (Conversación. Aura Rosmira y Stella Vida. Popayán, 2015); la Asociación Cultural de Palenque y el grupo Joven Internacional José Prudencio Padilla. Así como la vivencia de experiencias racistas, estas colectividades estudiantiles también tuvieron en común la relación que establecieron con Manuel Zapata Olivella. Según los testimonios, en varias oportunidades Manuel participó de espacios o encuentros con estas agrupaciones -con excepción del Frente Amplio por la Liberación del Negro Colombiano en la Universidad del Valle- para discutir asuntos de la identidad negra. 
Rosalba Castillo, lideresa afrocolombiana quien participó en uno de los espacios en los que Manuel Zapata Olivella presentó sus ideas sobre la trietnicidad, comentó lo siguiente:

Ese evento fue en el [75], en Cali, en la casa de un amigo y luego nos reunimos en obras públicas en la 4ta aquí en Cali. [...] Él hizo la presentación y habló de las trietnias, empezó a decir que éramos una mezcla de los negros, los mestizos, los indios, que él era la demostración, él era mulato, era la demostración, pero que había mucha discriminación (Conversación. Rosalba Castillo. Cali, 2015).

A diferencia de lo que muchos académicos pasan por alto ${ }^{4}$, el movimiento de la negritud liderada por el martiniqués Aimé Césaire, el guayanés Léon Damas y el senegalés Léopold Sédar Senghor tuvo un impacto muy importante en el continente suramericano ${ }^{5}$. Particularmente en Colombia, las esferas públicas afrocolombianas surgieron entre las décadas del setenta y el ochenta, como he señalado en mi tesis doctoral The negritude movements in Colombia. Manuel Zapata Olivella fue uno de los protagonistas principales que ayudó a consolidar esta corriente de pensamiento en el país.

El anterior testimonio es muy importante para entender el modo en que se configuró el contrapúblico afrocolombiano en los años setenta en Colombia como un terreno ideológicamente disputado. Durante este periodo, los países de Latinoamérica vivieron una "súbita escalada de movilizaciones con contenidos raciales", siendo Brasil uno de los países con mayor proceso de organización social y política (Andrews 297), lo que permitió la circulación de discursos tales como el poder negro, los derechos civiles, la nación musulmana liderada por Malcolm

4 Por ejemplo, Andrews considera que las movilizaciones políticas afro-latinoamericanas fueron de estudiantes y profesionales que "se unieron en los setenta y los ochenta para crear organizaciones latinoamericanas análogas al movimiento por los derechos civiles de Estados Unidos" (295), desconociendo la influencia del movimiento de la negritud en la región sur americana.

5 Sus planteamientos del lugar simbólico y político de África y sus descendientes revalorizaron la imagen del continente africano, su cultura, historia y descendencias desde una perspectiva universal y humanista. Así, redescubrieron África para los africanos y sus descendientes más allá de las imágenes racializadas popularizadas por Europa (Rabaka). 
$\mathrm{X}$, los discursos por la liberalización de los países africanos del dominio colonial europeo, la négritude, y la radicalización de la izquierda con la emergencia de grupos armados en Colombia, la revolución cubana y la intervención estadounidense en la región. La emergencia, entonces, de nuevas agrupaciones y colectividades estudiantiles se dio con el surgimiento de nuevas tendencias ideológicas que disputaron el terreno que el movimiento de la negritud estaba ganando con el papel desempeñado por intelectuales, entre ellos Manuel Zapata Olivella, y organizaciones afro. Por ejemplo, el Frente Amplio por la Liberación del Negro Colombiano de Cali, la Asociación Cultural de Palenque y la Minga del norte del Cauca, tuvieron una fuerte influencia del discurso marxista; mientras que Soweto fue influenciada por los discursos del poder negro, la izquierda colombiana y la lucha en contra del apartheid en Sudáfrica. Por último, el colectivo de Popayán, el grupo Joven Internacional José Prudencio Padilla, el Consejo Nacional de la Población Negra Colombiana liderado por Valentín Moreno, el partido político negro, el Movimiento de las Negritudes y el Mestizaje de Colombia y el Centro para la Investigación de la Cultura Negra en Colombia fueron fuertemente influenciados por el movimiento de la negritud. A diferencia del movimiento de la negritud francófona, el movimiento que se dio en Colombia y Latinoamérica no fue exclusivamente literario. Estos involucraron procesos de organización social y política electoral (Valderrama, The negritude movements 222; Wagbou et al. 108).

Muchos fueron los caminos que llevaron al arribo del discurso de la negritud a Colombia. Uno de ellos se gestó en las dinámicas colectivas que dieron paso a la conformación del grupo José Prudencio Padilla. Cuenta Nino Caicedo, quien fuera integrante del grupo y posteriormente miembro activo del Centro de Investigación Cultural liderado por Amir Smith Córdoba, que:

Nosotros estudiamos en Bogotá [...], allá convivimos con Amilkar Ayala y por medio de Amilkar, quien había llegado primero y tenía relación con los Olivella, no tanto con Manuel sino con Juan, con David Sánchez Juliao, con el doctor Tufit Meluk Aluma, con Amir Smith Córdoba. Amílcar me lleva a donde ellos [...], yo me quedé como alumno de los dos; con Tufit Meluk y Amir Smith. Con Meluk eran tardes de lectura; nos leía libros en francés, nos leía Negros son los dioses de mi África. En ese libro nos mostraba toda la importancia de la cultura que 
había existido y se había creado en África antes de que fuera occidentalizada, nos leía libros sobre la parte de la raza negra de Simón Bolívar. Nos hablaba de la abuela negra de Simón Bolívar (Conversación. Nino Caicedo, Cali, 2016).

Entre quienes participaron de esta colectividad se encuentran Eduardo Díaz Saldaña, Arturo Bobb Rodríguez, Amilkar Ayala, Tufit Meluk Aluma, Amir Smith Córdoba, Nino Caicedo, Leonor Murrillo, Manuel Zapata Olivella, entre otros. Mientras el sirio-libanés Tufit Meluk Aluma socializaba y discutía los contenidos de la revista Presencia Africana, creada por el movimiento de la negritud, el escritor y novelista Arnoldo Palacios aportaba desde la experiencia que tuvo personalmente con este movimiento ${ }^{6}$. Según mi conversación con el intelectual Tiberio Perea, "Arnoldo Palacios en ocasiones participó; él tenía un apartamento en la Calle 21 con Carrera 8va. Ahí nos reunimos, por ejemplo" (Conversación. Tiberio Perea. Bogotá, 2015). Del mismo modo, para 1974, Manuel Zapata Olivella ya había establecido contacto transnacional con figuras claves del movimiento de la negritud. El mismo Perea cuenta que participó del Coloquio de la Negritud y la América Latina, en Dakar, organizado por Léopold Sédar Senghor en 1974, siendo este último presidente de la República del Senegal. En este coloquio, se discutió particularmente la relación entre la negritud y el indigenismo (Zapata, "Negritud" 57-65).

A esta dinámica de tertulia se unirían posteriormente estudiantes afroamericanos que llegaron a Colombia en 1973, a través de becas académicas e intercambios otorgados por la Fundación Fulbright. Del encuentro entre afrocolombianos y afroamericanos se creó una esfera afrodiaspórica trasnacional, llamada "Entendimiento Mutuo". El intelectual afroamericano Laurence Prescott, en un intercambio de correos electrónicos, me expresó lo siguiente:

Los eventos sin mucha difusión en que participamos el profesor [David] Gilliam y su esposa [Bonita Gilliam], algunos compatriotas nuestros y yo, al lado de varios jóvenes colombianos, los bautizamos

6 Para entonces, Arnoldo Palacios había tenido la oportunidad de reunirse con intelectuales negros del movimiento de la negritud, como Aimé Césaire, Léon Damas, Léopold Sedar Senghor y Frantz Fanon. Así mismo, según su propio testimonio, participó del Congreso de Escritores Negros realizado en París (Conversación. Arnoldo Palacios. Bogotá, 2015). 
“Entendimiento Mutuo." Además de Arturo Rodríguez Bobb, algunos otros colombianos cuyos nombres me vienen a la mente son Doris García Mosquera, Eduardo Díaz, Leonor y Luis Murillo y Amilkar Ayala. Nos reunimos de vez en cuando para fomentar una mayor comprensión entre nosotros como personas de ascendencia africana. Nuestras conversaciones trataron de problemas y cuestiones particulares a nuestros respectivos pueblos y relevantes a las poblaciones de Afroamérica en general. Discutimos en grupo o individualmente las ideas de Malcolm X, Martin Luther King Jr., Frantz Fanon y otros. Aprendimos los nombres de Diego Luis Córdoba, Natanael Díaz y otros líderes afrocolombianos. Recuerdo que estuvo presente una vez el Dr. Manuel Zapata Olivella, quien nos dio una verdadera lección iluminadora sobre cómo los revolucionarios criollos (e.g., Bolívar) derrotaron y reemplazaron a los espańoles, pero dejaron de hacer grandes cambios políticos, sociales y económicos a favor de las masas subordinadas (negros, indígenas, mestizos). Creo que nos reunimos alguna vez para escuchar música y bailar" (Comunicación personal. Laurence Prescott, 2015).

Como resultado de este diálogo afrodiaspórico, se propuso la realización de la Primera Semana de Cultura Negra, en 1975, en la Biblioteca Nacional de la ciudad de Bogotá. La jornada de seis días se desarrolló del modo y con los participantes que se detallan a continuación 7 . El primer día, lunes 9 de junio, se presentaron "objetivos, generalidades, observaciones del movimiento Joven Internacional”, también hubo una sesión de "canciones negras", interpretadas por Nenfalia, Fredy García, Leonor y Nicolás Murillo y Alfonso Córdoba. El martes 10 de septiembre se organizó una "mesa redonda sobre concepto de la negritud", en la que participaron Tufit Meluk Aluma, Luis Vidales, Regelio Castillo Candelo, Jaime Quijano Caballero, Henry Luque Muñoz, Alfonso Mina Balanta, Jean Paul, Manuel Zapata Olivella y Nina de Friedemann. El moderador de la mesa fue Laurence Prescott. El miércoles 11 de junio se presentaron "Reseña histórica del folklore negro colombiano", por Delia Zapata Olivella, y la obra de Aimé Césaire por miembros del movimiento joven internacional. El jueves 12, se presentó "Problemáticas del negro en Colombia" por Daniel Santacruz,

7 El cronograma de actividades fue cedido muy amablemente por el intelectual y activista de la organización Joven internacional, Arturo Rodríguez Bobb. 
Ricaurte Palacios y Eduardo Díaz Saldaña. Ese mismo día se presentó un sociodrama por los miembros del movimiento joven internacional. El viernes 13, se realizó la conferencia "Breve análisis del panorama mundial del negro” por Arturo Rodríguez Bobb y Amilkar Ayala. En el mismo día se abrió una sesión de "cuentos negros" y un "recital poético" por Sebastián Salgado. Finalmente, el sábado 14 de junio se programó "Sentimiento negro en la expresión de su canto", en el que intervinieron Amilkar Ayala, Leonor Murillo, Nenfalia, Fredy García, Nicolás Murillo, Sebastián Salgado y Catalino del Manglar.

Tal como lo hizo en la Primera Semana de Cultura Negra, Manuel Zapata Olivella participó en muchos otros espacios de discusión y socialización del discurso de la negritud o de la cultura negra. Baudilio Revelo, representante de la Asociación de Colonias del Pacífico en Cali por muchos años, recuerda que "Manuel Zapata Olivella organizaba tertulias, pero no sé cómo era, era de negritud porque en esa época ya se estaba presentando movimientos de derechos civiles en los Estados Unidos" (Conversación. Baudilio Revelo. Cali, 2016). Algunos de los espacios más importantes en los que Manuel Zapata Olivella participó fueron los Congresos de la Cultura Negra de las América, realizados en Cali (1977), Panamá (1980) y Brasil (1982). Mientras que en los congresos de Panamá y Brasil Manuel Zapata Olivella participó como coordinador internacional, en Cali fue su presidente. Fue el encargado de convocar a las delegaciones de Colombia ${ }^{8}$, África, el Caribe y Norteamérica'. Así mismo, dio el discurso de apertura llamado "Una nueva era para la identidad de América", en el que propuso una identidad latina en la que "la identidad del negro" y sus "descendientes africanos" fueran reconocidas por el "sufrimiento de las postergaciones heredadas del

8 Entre los 63 colombianos que participaron se encuentran los afrocolombianos y afrocolombianas Rosalba Castillo, Carlos Calderón del Chocó, Jorge Artel y Aquiles Escalante, del Caribe Colombiano; Helcías Martán Góngora y Agustín Revelo, de Guapi; Marino Viveros Mancilla, del Norte del Cauca, y quien participara del Día del Negro; Delia y Manuel Zapata Olivella, Rosa Bosch, esposa de Manuel Zapata y su sobrino Alexis Zapata, quien fuera miembro del grupo de trabajo negro en la Universidad de Popayán. Algunos intelectuales blanco-mestizos que participaron fueron Nina S. de Friedemann, Nancy Motta y Jaime Arocha. Estos antropólogos serían claves luego en la reforma constitucional (Agudelo 179).

9 Angola (1); Brasil (6); Costa Rica (1); Chile (1); Ecuador (4); Egipto (1); España (1); Estados Unidos (23); Honduras (3); México (1); Nigeria (3); Panamá (8); Perú (2); Puerto Rico (1); Senegal (3); y representantes de la Unesco y la OEA. 
sistema esclavista" (Zapata, "Discurso de apertura" 3). Para Andrews, esto hace parte del proceso de ennegrecimiento de la región (295). Así, el continente fue concebido ya no en términos de la ideología del mestizaje ni, mucho menos, del binarismo latino-anglosajón que negó la presencia afrodescendiente. La identidad continental americana se propuso sobre la base de las experiencias negras.

La importancia de estos tres congresos es innegable, pues permitieron la discusión de lo que significa ser negro en el continente americano desde las diferentes posturas ideológicas señaladas anteriormente. Revisemos algunos de los resultados obtenidos del Primer Congreso de la Cultura Negra, liderado por Manuel Zapata Olivella. La comisión de etnia negra ${ }^{10}$ y mestizaje definió que una identidad étnica es "cuando un individuo es señalado como negro y cuando él se siente negro [...], esta identidad es fundamentalmente de una Etnia Negra" (Comisión de Etnia Negra y Mestizaje 145). Discriminación racial es "cuando un individuo o un grupo que no es negro y que ejerce el poder para negarle recursos económicos, educativos, políticos o religiosos a un negro o a un grupo de negros" (ibid.). También declaró que "en América Latina se práctica la discriminación racial de manera solapada, sutil, abierta o encubierta. Tal discriminación utiliza las diferentes tonalidades del color epidérmico del negro como mecanismo para lograr que el hombre negro desaparezca a través de la ideología del blanqueamiento" (ibid.). Esta última definición se diferencia de la forma en que se entiende el racismo para el caso de los Estados Unidos. El informe dice que "cuando la discriminación racial está regulada por leyes e instituciones de un país, estamos frente al ejercicio del racismo. Aunque en los Estados Unidos ciertas leyes [...] del racismo se abolieron, [...] se ejerce la discriminación racial a nivel privado e institucional de una manera que pretende ser encubierta, y que continúa protegida por la ley" (ibid.). La comisión estableció que los problemas sufridos por descendientes africanos de las Américas no se resuelven con reformas de naturaleza utópica, "sino que se necesitará un cambio básico estructural de la sociedad y del sistema

10 En adelante señalaré en cursiva aquellas palabras y conceptos usados en el Congreso y que en la actualidad muchos consideran que son de uso exclusivo del movimiento social afrocolombiano contemporáneo. Mi intención es puntualizar aquellos términos y referencias que fueron usados previamente a la constitución de 1991, la ley 70 de 1993 y el multiculturalismo estatal, para establecer su importancia desde entonces. 
económico-político vigente" (147). Lo anterior, debido a que el "sistema capitalista difunde la ilusión de igualdad étnica, pero en la práctica ejerce acciones discriminatorias al no dar oportunidades similares a las capas negras e indígenas" (Comisión Creatividad Social y Política 156) ${ }^{11}$.

Quisiera destacar la discusión suscitada acerca del concepto de "negritud", ya que refleja las tensiones y debates generados en el congreso. El informe registra quese ha señalado que, aunque la Negritud afirma que todos los negros son parte de una familia y pueden tener un sitio en África, no se trata de una vuelta en masa al continente africano, si no de la identificación con la cultura negra y la lucha en los diversos países donde el negro esté presente, para defender esta identificación y esta cultura (Comisión de Etnia Negra y Mestizaje 147).

Por otro lado, se señaló "la existencia de una manipulación de los elementos de la cultura negra-africana por parte de las sociedades dominantes blancas como instrumento de dominio y sujeción" (ibid.). Por lo cual fue necesario aprobar la siguiente declaración: "Se considera la Negritud como una estrategia alternativa de participación y reclamo de derechos en Colombia y en los otros países asistentes al Congreso" (ibid.). Por ello, la Comisión de Creatividad Social y Política expresó que -aun cuando "se exalta el movimiento de la negritud iniciado en 1932 por los intelectuales negros Leopoldo Sedar Senghor, Aimé Césaire y León Damás"- "los negros afroamericanos necesitan crear su propia ideología en las luchas de la liberación cultural [y] en su lucha contra la opresión colonial" (156).

De la Comisión de Filosofía y Efectividad destacamos lo siguiente: "El trabajo esclavo negro tuvo una importancia decisiva para el enriquecimiento de los blancos europeos y criollos durante la Colonia. Este proceso fue común a los EE.UU. y a América Latina” (151). Con la abolición de la esclavitud el estatus del negro pasó de ser esclavo a ser definido por su inferioridad social y económica en la vida republicana (ibid.). Ha habido transformaciones meramente en el plano formal, se decía, pero en la práctica "la población descendiente del África negra

11 En este punto quisiera señalar cómo el congreso articula discursos de clase y raza para explicar la situación económica y social de las comunidades negras, aspectos que lo diferencian del movimiento de la negritud francófono. 
continúa en una situación de dependencia y paternalismo" (ibid.). Así, "la correlación existente entre raza y clase social ha impedido que el negro identifique con claridad las razones de la discriminación social de la cual es víctima" (ibid.). Es importante señalar el reconocimiento hecho al papel histórico de las mujeres negras en las luchas sociales, culturales y políticas de las comunidades negras y la doble opresión que sobre ellas recaen. La comisión manifiesta que se debe reconocer el "importante papel histórico de la esclava negra en su continua lucha por la libertad, como también en asegurar la supervivencia de su raza" (151). Además, se pide a todos "los hombres negros de todo el mundo reconocer que el logro de un mundo renovado [...] depende en gran parte de la liberación de la mujer negra, del peso impuesto por el estereotipo doble, que circunscribe su existencia a las funciones de objeto sexual y reproductor" (ibid.). Como se puede observar, las discusiones en los espacios en los participó Manuel Zapata Olivella se dieron bajo disputas ideológicas de diferentes fuentes de movilización política, lo que trajo consigo tensiones y conflictos ideológicos sobre la identidad afrocolombiana.

\section{Un MESTIZAJE CONTRAHEGEMÓNICO CUESTIONADO}

La presencia de Manuel Zapata Olivella en eventos públicos produjo discusiones, tensiones y conflictos entre su perspectiva y la de sus interlocutores. Bien es sabido que para Manuel Zapata Olivella la identidad racial afrocolombiana tuvo su fundamento mestizo -autores como Arboleda, Caicedo, Viveros y Valderrama así lo han señalado-. Es sabido también que su idea de mestizaje presentó muchas contradicciones, confusiones y ambigüedades por corresponder al término usado para negar la existencia de una cultura negra y del racismo en Colombia (Viveros 465). Sin embargo, no podemos negar que su planteamiento sobre el mestizaje fue también una idea radical (Arboleda 230) en la medida que, contraria a la versión hegemónica, su idea del mestizaje afirmó la presencia física, biológica y cultural negra como condición necesaria de la identidad nacional colombiana. De esta forma, Manuel Zapata Olivella emprendió una lucha titánica por la inclusión de lo negro y el reconocimiento de su participación en el proceso de construcción 
de "[una] nación [mestiza radical], en un contexto social que hacía la glorificación de lo mestizo [...] que apuntalaba un discurso jerárquico de lo blanco, discriminando lo indígena y lo negro" (Viveros 465). Como se ha podido observar hasta aquí, el recorrido hecho muestra una clara agenda intelectual, cultural y política de lucha contra el racismo en Colombia.

A pesar del reconocimiento y liderazgo de Manuel Zapata Olivella, sus planteamientos generaron muchas controversias, precisamente por sus ideas sobre el mestizaje triétnico o por su proyecto cultural y folclórico. Por ejemplo, los colectivos estudiantiles Soweto y La Minga, y organizaciones como el Consejo Nacional de la Población Negra Colombiana y el Centro para la Investigación de la Cultura Negra se mostraron reacios a sus planteamientos. Estas agrupaciones cuestionaron duramente su valor libertario y emancipador por su falta de claridad y precisión. Una de las críticas al proyecto cultural de Manuel tuvo lugar en los Seminarios Sobre Formación y Capacitación de Personal Docente en Cultura Negra, organizados por el Centro para la Investigación de la Cultura Negra en Colombia, entre 1978 y 1983.

Particularmente, me refiero al seminario IV, celebrado en Valledupar en 1981. Sus "participantes [...] cuestionaron los objetivos del 'Centro para la investigación de la cultura negra', entidad organizadora del seminario" ("Éxito en el seminario de cultura negra" 6a). Según lo reporta el periódico El Tiempo, "[e]l certamen cobró acalorada discusión cuando Manuel Zapata Olivella entró a defender, en nombre del 'cientificismo abstracto', el mestizaje negro: 'quién puede impedir que yo defienda la parte del blanco que hay en mí” (ibid.). El africano N'dong Ondo Andeme se encontraba en el seminario y, según la plantea el periódico, "se pronunciaría para que el público comprendiera cómo puede hacer daño la falta de identidad" (ibid.). Como resultado de la discusión, el seminario decretó la necesidad de orientar estudios demográficos de "la población negra colombiana y presentó la importancia de tener en cuenta quién es realmente negro en una sociedad donde todos quieren ser blancos" (ibid.). Así mismo, Juan de Dios Mosquera, líder de la organización Cimarrón, expresaba al respecto que los hermanos Zapata Olivella "reivindicaban la población y la cultura que había sido mestizada, blanqueada, pero el blanqueamiento es un aspecto político que no tiene que ver con el color de la piel” (Conversación. Juan De Dios Mosquera. Bogotá, 2015). 
Las críticas anteriores dejan ver el disputado campo ideológico que se presentó en la década de los setenta en Colombia. Claramente, el africano N'dong Ondo Andeme y Juan de Dios Mosquera se paran desde corrientes ideológicas (probablemente, el panafricanismo y el poder negro) que reivindican la centralidad de lo negro por encima de las mezclas raciales y el mestizaje. $Y$ es que, a pesar de la política de la realización -entendida como los proyectos intelectuales afrodescendientes cultivados y movilizados con la esperanza de una sociedad capaz de cumplir, para todas las razas, los ideales de la modernidad (Gilroy 37)-, que agenció Manuel Zapata Olivella, no podemos desconocer que su proyecto intelectual y cultural alrededor del mestizaje fue confuso para explicar cómo un ser humano de tonalidad de piel negra fuera un mestizo. Esta confusión se dio, por un lado, debido al momento histórico en el que Manuel Zapata Olivella propuso su idea de mestizaje. Era un contexto dominado por la idea oficial de mestizaje, que buscó blanquear racial y culturalmente a la sociedad colombiana. Es decir, eliminar la presencia de personas negras, negándolas bajo la idea de que todos somos mestizos. Así, la propuesta de Manuel Zapata Olivella no logró diferenciarse de la mirada oficial. Por otro lado, su propuesta conflictuó con las propuestas afrocéntricas -por ejemplo, el poder negro, el panafricanismo, la negritud, etcétera-, que posicionaron una identidad negra heredera de África y en la que el mestizaje no era relevante políticamente. De esta forma, aunque su política de la realización buscó reconocer las mutuas influencias que existen entre los grupos racializados más allá de los binarismos de la modernidad, su propuesta no pudo superar estas ambigüedades: "El planteamiento como concepto sociológico [y cultural] es diferente al blanqueamiento en términos raciales, son dos cosas diferentes; pero que confunden a nuestro pueblo" (Conversación. Juan De Dios Mosquera. Bogotá, 2015).

A lo anterior se suma que su proyecto fue excluyente de los sectores populares negros/afrocolombianos. Así me lo contaba José Licer Mosquera: "Una vez le dije, su trabajo está muy elitista [...], la época exige que hagamos un trabajo más colectivo, [...] además, ir al pueblo, pues él se centró a dar conferencias en universidades, y de la élite" (Conversación. José Eulices Mosquera, Medellín, 2016). El proyecto de Manuel Zapata Olivella tenía como público a aquellos que en los centros urbanos podían leer, comprar sus libros, tener radio o televisión para escuchar u oír sus programas, y recursos para asistir a las conferencias o congresos 
intelectuales organizados por él. Probablemente sin pensarlo, Manuel Zapata Olivella terminó reproduciendo prácticas de subordinación contra la propia población que defendía: "En Bogotá [...] más o menos pude comprar algunas de las obras de él, pero eso lo compraba la intelectualidad básicamente blanca [...]; los poquitos [libros] que quedaban en Colombia los conseguían la élite y por el mismo costo que vendían esas editoriales" (Conversación. José Licer Mosquera, Medellín, 2016). Al final de los años setenta, el reclamo de las nacientes organizaciones como La Minga, con una agenda mucho más rural y campesina, criticaron que "ellos no tenían trabajo de campo [...]. Nunca se organizaron para exigir la recuperación de la tierra” (Conversación. Guillermo Mina. Villa Rica, Norte del Cauca, 2015).

\section{Comentarios finales}

Con el presente escrito he querido mostrar la trayectoria intelectual de Manuel Zapata Olivella en el proceso organizativo afrocolombiano, con el propósito de posicionarlo como un intelectual comprometido, que no solo aportó en la construcción de una literatura revolucionaria o de combate, sino que también avanzó en acciones concretas que favorecieron la organización social y política contra la opresión. En este orden de ideas, el escrito muestra la manera en que Manuel Zapata Olivella se relacionó con organizaciones, agrupaciones e intelectuales afrocolombianos para discutir sobre asuntos relacionados con la identidad negra y el racismo en Colombia. Como se observó a través del texto, Manuel Zapata Olivella fue un actor clave en la dinamización de las esferas públicas que llevaron a la conformación del contrapúblico afrocolombiano en los años setenta. Así mismo, queda claro que su proyecto cultural y político no estuvo ausente de cuestionamientos y críticas por su limitada y contradictoria interpretación de la identidad triétnica. Estas tensiones dan cuenta, entonces, de intentos de definirse y redefinirse negro/afro-subalternos en los que los planteamientos de Manuel Zapata Olivella fueron dinamizadores de la discusión y debates.

Espero que el presente trabajo sirva como un modelo inicial que nos permita estudiar a la intelectualidad afrodiaspórica en Latinoamérica teniendo como base sus dimensiones políticas: el pensamiento crítico y 
militante. En este escrito me centré solo en la segunda de ellas, dada la preeminencia de los estudios que abordan la primera. Observando estas dimensiones en la trayectoria intelectual de Manuel Zapata Olivella, podemos sostener que guarda mucha similitud con las trayectorias intelectuales de figuras tan importantes en el mundo negro/afrodescendiente como W.E. B. Du Bois, C. L. R. James, Walter Rodney y Frantz Fanon, entre otros -guardando, por supuesto, sus diferenciaciones ideológicas y radicalismo político-. Lo importante es reconocer las posibilidades de abrir estudios y reflexiones que nos permitan apreciar la participación política de los intelectuales afrocolombianos y afrolatinos.

\section{BiBLIOGRAFÍA}

Agudelo, Carlos. Multiculturalismo en Colombia: Política, inclusión y exclusión de poblaciones negras. Medellín, Carreta Editores, 2005. Angulo, Alberto. Moros en la costa: Vivencia afrocolombiana en la cultura colectiva. Bogotá, Docentes Editores, 1999.

Andrews, G. R. Afro-Latinoamérica, 1800-2000. Madrid, Iberoamericana, 2007.

Arboleda, Santiago. Le han florecido nuevas estrellas al Cielo. Suficiencias Intimas y Clandestinización del pensamiento Afrocolombiano. Cali, Poemia, 2016.

Caicedo, José. A mano alzada. Memoria escrita de la diáspora intelectual afrocolombiana. Popayán, Sentipensar, 2013.

Collazos, Óscar. “Un clásico afroamericano” (Prólogo). Las estrellas son negras, Arnoldo Palacios, Bogotá, Ministerio de Cultura, 2010.

Comisión de Creatividad Social y Política. "Creatividad Social y Política". Primer Congreso de la Cultura Negra de las Américas, Cali, Colombia: Actas, Fundación Colombiana de Investigaciones Folclóricas, Bogotá, Unesco/Fundación Colombiana de Investigaciones Folclóricas/ECOE, 1988, pp. 155-157.

Comisión de Etnia Negra y Mestizaje. "Etnia Negra y Mestizaje". Primer Congreso de la Cultura Negra de las Américas, Cali, Colombia: Actas, Fundación Colombiana de Investigaciones Folclóricas, Bogo- 
tá, Unesco/Fundación Colombiana de Investigaciones Folclóricas/ ECOE, 1988, pp. 154-158.

Comisión de Filosofía y Afectividad. "Filosofía y Afectividad". Primer Congreso de la Cultura Negra de las Américas, Cali, Colombia: Actas, Fundación Colombiana de Investigaciones Folclóricas, Bogotá, Unesco/Fundación Colombiana de Investigaciones Folclóricas/ ECOE, 1988, pp. 151-153.

Comisión Material y Artística. "Creatividad Material y Artística”. Primer Congreso de la Cultura Negra de las Américas, Cali, Colombia: Actas, Fundación Colombiana de Investigaciones Folclóricas, Bogotá, Unesco/Fundación Colombiana de Investigaciones Folclóricas/ ECOE, 1988, pp. 159-163.

Dawson, Michael. Black Visions: The Roots of Contemporary African-American Political Ideologies. Chicago, University of Chicago Press, 2001.

De Friedemann, Nina S. "Estudios de negros en la antropología colombiana: Presencia e invisibilidad". Un siglo de investigación social: Antropología en Colombia, Nina S. de Friedemann, Jaime Arocha y Xochitl Herrera (eds.), Bogotá, Etno, 1984, pp. 155-172.

"Éxito en el seminario de cultura negra". El Tiempo, Bogotá, 20 de octubre de 1981, p. 6 a.

Fraser, Nancy. "Rethinking the Public Sphere: A Contribution to the Critique of Actually Existing Democracy”. Social Text, N²5/26, 1990, pp. 56-80. Web. Disponible en: https://doi.org/10.2307/466240

Fundación Colombiana de Investigaciones Folclóricas (ed.). Primer Congreso de la Cultura Negra de las Américas, Cali, Colombia: Actas. Bogotá, UNESCO/Fundación Colombiana de Investigaciones Folclóricas/ECOE, 1988.

Karabalí, Manuel. "Estudio del negro en Colombia". Cromos, $\mathrm{N}^{\circ}$ 64, 1947, p. 9.

Lasso, Maritza. Myths of Harmony: Race and Republicanism during the Age of Revolution, Colombia 1795-1831. Pittsburgh, PA, University of Pittsburgh Press, 2007.

Lewis, Marvin. A. Treading the Ebony Path: Ideology and Violence in Contemporary Afro-Colombian Prose Fiction. Columbia, University of Missouri Press, 1987. 
Pisano, Prieto. Liderazgo politico "negro" en Colombia, 1943-1964. Bogotá, Universidad Nacional de Colombia, 2012.

Prescott, Laurence. Without Hatreds or Fears: Jorge Artel and the Struggle for Black Literary Expression in Colombia. Detroit, Wayne State University Press, 2000.

"Brother to Brother: The Friendship and Literary Correspondence of Manuel Zapata Olivella and Langston Hughes". Afro-Hispanic Review, vol. 25, № 1, 2006, pp. 87-103.

Valderrama, Carlos. "Folclore, raza y racismo en la política cultural e intelectual de Delia Zapata Olivella. El campo político-intelectual Afrocolombiano". Revista CS, N¹2, 2013, pp. 259-96. Web. Disponible en: https://doi.org/10.18046/recs.i12.1674

The negritude movements in Colombia. Tesis para obtener el grado de Doctor en Filosofía, Amherst, Universidad de Massachusetts, 2018. Web. Disponible en: https://scholarworks.umass. edu/dissertations_2/1408/

Viveros, Mara. "Manuel Zapata Olivella 1920-2004". Pensamiento Colombiano del siglo XX, Tomo III. Carmen Millán de Benevides, Santiago Castro Gómez y Guillermo V. Hoyos (eds.), Bogotá, Universidad Javeriana, 2013, pp. 465-500.

Wabgou, Maguemati et al. (eds.). Movimiento social afrocolombiano, negro, raizal y palenquero: el largo camino hacia la construcción de espacios comunes y alianzas estratégicas para la incidencia politica en Colombia. Bogotá, Universidad Nacional de Colombia/Instituto Unidad de Investigaciones Jurídico-Sociales Gerardo Molina, 2012.

Zapata Olivella, Manuel. "Discurso de Apertura a Cargo del Doctor Manuel Zapata Olivella". Primer Congreso de la Cultura Negra de las Américas, Cali, Colombia: Actas, Fundación Colombiana de Investigaciones Folclóricas, Bogotá, Unesco/Fundación Colombiana de Investigaciones Folclóricas/ECOE, 1988, pp. 19-21.

- ¡Levántate mulato! Por mi raza hablará el espíritu. Bogotá, Rei Andes, 1990.

"Negritud, Indianidad y Mestizaje en Latino América". Présence Africaine, $\mathrm{N}^{\circ} 145,1988$, pp. 57-65. 